\title{
溶解腐殖质在云母界面上吸附聚集行为 的原子力显微镜观察 ${ }^{*}$
}

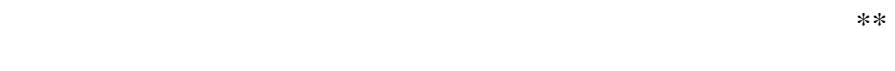 \\ (1) 中国科学院生态环境研究中心环境水质学国家重点实验室, 北京 100085; \\ (2) 北京交通大学土木建筑工程学院, 北京 100044)
}

\begin{abstract}
摘要 腐殖质作为天然颗粒物有机质的主要成分, 其结构形态直接影响有机污染物在天然颗 粒物表面上的吸附、解吸以及在环境中的迁移、传输性能等一系列环境行为和过程. 对官厅和天 津两种不同来源和组成的腐殖质样品在云母界面上的吸附聚集行为进行了原子力显微镜成像观 察. 结果表明, 官厅腐殖质在云母界面上呈不规则链环结构, 链平均宽度为 $40 \mathrm{~nm}$; 而天津腐殖 质则呈致密的球形颗粒, 直径为 250 330 nm. 在不同浓度条件下, 两种腐殖质可通过界面自组装 形成多孔片层、球形颗粒聚集体、疏松海绵状结构、支链及条带状致密晶态有机质等多种界面聚 集体形态. 与天津腐殖质相比, 官厅腐殖质的聚集体结构较疏松, 且结构形态变化更丰富, 这可 能与官厅腐殖质中含有较多的极性物质有关. 有萗共吸附条件下官厅腐殖质在云母界面上的支 链状结构明显变窄, 呈多孔片层状的局部放大结构变得更加致密, 孔结构趋向减少, 并同时观察 到有凝胶团块状结构出现, 表明萗分子与溶解腐殖质分子发生了相互作用, 并使腐殖质分子之间 的联系进一步加强, 结合更加紧密. 同时, 腐殖质多孔链环结构的中心有可能作为疏水性吸附区 域与萗分子发生相互作用, 从而促进了萗在天然颗粒物界面上的吸附和聚集.
\end{abstract}

\section{关键词原子力显微镜(AFM) 腐殖酸 微观形貌 聚集行为 吸附}

腐殖质是一类广泛存在于土壤、沉积物及各种天 然水体环境中, 或者通过物理、化学等手段从中提取 出来, 颜色呈黄色或深褐色的天然有机化合物, 其来 源、组成和结构十分复杂, 分子量一般可从数百到上
百万 ${ }^{[1,2]}$. 通常认为, 腐殖质是由动植物及微生物残 体(木质素等生物高分子)在天然环境中经生物酶分 解、氧化以及微生物合成等不同反应阶段和过程逐步 演化而成的具有多分散结构特征的有机高分子混合 
物 $^{[3]}$. 由于腐殖质本身在物理、化学性质上表现出一 定条件下的非均相性以及其分子结构上的复杂性, 它们既可溶解或悬浮在水体中, 又可在溶液中通过 弱相互作用等形成分子缔合物, 并在界面上聚集形 成各种自组装结构 ${ }^{[4,5]}$, 因此, 腐殖质在一系列环境 过程和行为中发挥着十分重要的作用. 例如, 作为土 壤及沉积物中天然有机质的重要组成部分, 腐殖质 对疏水性有机污染物 (hydrophobic organic contaminants, HOCs)在天然颗粒物表面的吸附、解吸及其在 环境中的迁移、传输以至污染物的最终归宿等一系列 环境行为和过程起着决定性作用 ${ }^{[6 \sim 13]}$. 同时, 由富里 酸和部分腐殖酸组成的溶解腐殖质(dissolved humic substances)还作为天然水体中有机质的主要成分, 在 水体环境化学中起着重要作用, 它可以对水体中痕 量金属的化学形态进行修饰、通过在无机颗粒物表面 上的吸附作用使水体中悬浮颗粒变得更稳定 $[14,15]$ 以 及增强疏水性有机污染物在水体中的传输性能等 ${ }^{[16]}$. 因此, 观察腐殖质分子及其聚集体的微观形貌, 研究 其在固液界面上的吸附聚集行为对于深入认识腐殖 质在环境科学中的重要作用, 探索其在环境微界面 过程中的作用机理具有十分重要的意义.

有关腐殖质大分子的结构形态, 根据腐殖质在溶 液中所处的物理化学状态(包括浓度、溶液 $\mathrm{pH}$ 、离子强 度等) 不同, 已经提出具有聚电解质特性的柔性链模 型 ${ }^{[17]}$ 以及可形成致密球形颗粒的随机卷曲链环模 型 [18]等两种理论模型, 并已有一些相关文献通过扩散系 数的测定 $[18,19]$ 、分子尺寸排阻色谱(size exclusion chromatography, SEC) ${ }^{[3]}$ 、发光/荧光光谱(luminescence spectroscopy) $)^{[20]} 、{ }^{13} \mathrm{C}$-核磁共振(NMR) ${ }^{[21 ~ 23]}$ 和红外(IR) 光谱分析以及扫描电镜 ${ }^{[21,24]}$ 、透射电镜等对腐殖质分 子及其聚集体的特性进行了研究表征. 例如, Stevenson和 Schnitzer ${ }^{[25]}$ 对稀的腐殖质水溶液经快速冷 冻干燥沉积在云母表面上的富里酸及腐殖酸分子聚 集体的形状、大小及其聚集行为用透射电镜(TEM)进 行了研究, 并观察到随浓度的增加腐殖酸聚集体的 形貌由球状颗粒凝聚成纤维链状或加宽的丝带状结 构, 在高浓度下逐渐演变为多孔片层状结构. 近年来, 原子力显微镜的发明以及其在微孔、介孔材料表面或 界面表征方面具有的独特分辨能力 ${ }^{[26]}$, 又为人们在
纳米尺度上深入研究腐殖质分子在固液界面上的分 形聚集行为提供了一种强大的仪器技术手段. Heil和 Sposito ${ }^{[27]}$ 对含伊利石的土壤胶体及伊利石地质样品 等两种颗粒物的形貌及表面特征进行了AFM观察, 并对土壤有机质在颗粒物表面的修饰及颗粒物表面 粗糙抑制伊利石土壤胶体颗粒间的絮凝作用进行了 初步研究. Gerin及Dufrêne ${ }^{[28]}$ 最近也对 3 种天然土壤 颗粒物的表面结构进行了AFM成像观察, 并结合X射线光电子能谱对土壤颗粒表面的有机吸附层进行 了研究表征. 在经分离提取的腐殖质样品的微观结 构形态研究方面, 目前也有部分原子力显微镜的应 用文章 [29 36]报道，并观察到球状颗粒、海绵状结构、 支链状、多孔层片状以及环状结构等腐殖质聚集体 的形貌特征. 例如, Maurice等人 ${ }^{[29,30]}$ 对水溶液条件 下吸附到云母表面上的腐殖酸分子聚集体结构进行 了原位液态成像观察, 得到了呈数十纳米大小的环 状结构腐殖酸分子聚集体形貌. Wilkinson等 ${ }^{[31,32]}$ 对 水体中包括腐殖酸及多糖等有机生物大分子在内的 胶体颗粒用TEM及AFM分别进行了观察与表征, 并 就溶液 $\mathrm{pH}$ 及离子强度对两种不同来源和组成的腐殖 酸样品在云母表面上的吸附结构的影响进行了对比 研究, 观察到平均高度为 $0.5 \sim 2 \mathrm{~nm}$ 、呈小球颗粒状的 腐殖酸分子聚集体形貌. Plaschke等 ${ }^{[33}$ 35]对在不同 $\mathrm{pH}$ 及高价钶系金属铕离子 $\left(\mathrm{Eu}^{3+}\right)$ 存在条件下腐殖酸分 子在云母表面上的吸附聚集情况进行了液体环境 $\mathrm{AFM}$ 原位观察, 指出酸性 $\mathrm{pH}$ 条件及高价金属离子存 在可诱导腐殖酸分子在云母表面上发生聚集, 并观 察到扁平颗粒(直径 8 13 nm)、颗粒聚集体、长链状 结构及环形组装结构等聚集体形貌. Liu等 ${ }^{[36]}$ 对冷冻 及空气中自然干燥等两种样品制备方法所得到的腐 殖酸吸附层的厚度进行了对比测定. 刘燕等 ${ }^{[37]}$ 研究 了城市污水中有机底物的特性对活性污泥胞外聚合 物产生量及其组成的影响, 并通过扫描电子显微镜 和原子力显微镜对活性污泥的微观形貌进行了观测.

本文在天然沉积物质量评价研究项目工作基础 上，结合疏水性有机污染物在天然颗粒物界面上的 吸附机理研究, 对溶解腐殖质的界面吸附聚集行为 用原子力显微镜作了进一步的成像观测. 选择天然 云母作为模型表面, 对从官厅水库水体沉积物中提 
取的天然腐殖质以及市售天津产地的商品腐殖质等 两种不同来源及组成的腐殖酸样品在不同浓度条件 下的界面吸附聚集行为进行了研究，并对有菜共吸 附条件下官厅腐殖质界面吸附聚集行为的变化情况 进行了对比观测, 对其可能的作用机理进行了理论 解释.

\section{1 实验部分}

\section{1 实验材料与试剂}

实验用水为超纯离子交换水(电阻率为 $18 \mathrm{M} \Omega /$ $\mathrm{cm})$, 所用化学试剂均为分析纯, 氮气为普通氮气.

实验采用两种不同来源及组成的腐殖酸样品, 其中一种来自官厅水库水体沉积物(底泥), 由本实验 室依据国际腐殖质协会推荐的提取方法(IHSS) ${ }^{[38]}$ 进 行提取而得; 另外一种为市售商品腐殖酸，从煤中提 取而得, 产地来自天津.

官厅腐殖酸提取方法如下: 将一定量沉积物加 入到 $0.1 \mathrm{~mol} / \mathrm{L} \mathrm{NaOH}$ 溶液中, 通入氮气 $1 \mathrm{~h}$ 后密封, 于摇床上振荡 $12 \mathrm{~h}$, 离心, 倒出上清液, 残渣再加入 $\mathrm{NaOH}$ 溶液, 重复上述步骤两次, 合并上清液. 此上 清液中溶有大量腐殖酸, 用 $6 \mathrm{~mol} / \mathrm{L} \mathrm{HCl}$ 溶液调至 $\mathrm{pH}=2$, 使腐殖酸析出, 静置过夜, 离心并弃去上清液, 腐殖酸残渣用去离子水漂洗 3 次, 室温下自然晾干.

两种腐殖质样品的基本理化性能，其中包括总 有机碳(TOC)、溶解有机碳(DOC)、化学元素组成、 阳离子交换容量(cation exchange capacity, CEC)、 CPMAS ${ }^{13} \mathrm{C} N M R$ 及 ${ }^{1} \mathrm{H} N M R$ 光谱分析等见表 1 及表
$2^{[39]}$. 有机碳含量(TOC和DOC)采用Apollo-9000 TOC 分析仪(Tekman-Dohrmann公司，美国)进行测定，碳标 准溶液用邻苯二甲酸氢钾 $\left(115^{\circ} \mathrm{C}\right.$ 干燥 $\left.2 \mathrm{~h}\right)$ 进行配制. 元素分析采用高温燃烧法, 在Vario-EL型元素分析仪 (Elementar公司，德国)上进行测定．阳离子交换容量 采用文献[40]报道的 $\mathrm{BaCl}_{2}$ 法测定. CPMAS ${ }^{13} \mathrm{C} \mathrm{NMR}$ 及 ${ }^{1} \mathrm{H} \mathrm{NMR}$ (用 $\mathrm{D}_{2} \mathrm{O}$ 作溶剂)核磁共振光谱分析分别采 用Avance-DPV型 $300 \mathrm{MHz}$ (Bruker仪器公司, 德国. 测定条件: 探针直径 $4 \mathrm{~mm}$, 自旋速率 $13 \mathrm{kHz}$, 接触 时间 $2 \mathrm{~ms}$, 循环延迟时间 $1 \mathrm{~s}$, 扫描宽度 $27 \mathrm{kHz}(\delta=$ 368), 谱线展宽 $100 \mathrm{~Hz}$ )及Avance-DPX型 $500 \mathrm{MHz}$ (Bruker仪器公司, 德国. 测定条件: 探针直径 $5 \mathrm{~mm}$, 循环延迟时间 $1 \mathrm{~s}$, 扫描宽度 $8012 \mathrm{~Hz}(\delta=16)$, 谱线 展宽 $3.00 \mathrm{~Hz}$, 扫描次数 1000 次, 测定温度 $295 \mathrm{~K}$, 采 用 “水闸门” 技术以消除水峰干扰) 核磁共振光谱仪 进行测定.

荎储备液的配制：将过量的荎加入到由 0.005 $\mathrm{mol} / \mathrm{L} \mathrm{CaCl}$ 及 $100 \mathrm{mg} / \mathrm{L} \mathrm{NaN}_{3}$ 所组成的混合溶液中, 超声处理 $1 \mathrm{~h}$, 振荡过夜, 使之达到饱和溶解, 溶液用 GF/F 玻璃纤维滤膜(英国 Whatman 公司)过滤，清液储 存于棕色容量瓶中, 用封口膜密封, 在冰箱内保存.

天然云母片 $(10 \mathrm{~mm} \times 10 \mathrm{~mm})$ : 将长 $3 \mathrm{~cm}$, 宽 2 $\mathrm{cm}$, 厚度为 $0.18 \sim 0.20 \mathrm{~mm}$ 的市售商用天然云母长条 用剃须刀片切成 $1 \mathrm{~cm}$ 见方的正方形, 用去离子水淋 洗除去杂质颗粒，再用 $95 \%$ 乙醇洗涤，最后用无水乙 醇洗一遍，放到洁净干燥的培养皿中，室温晾干后， 保存备用。

表 1 两种腐殖质的基本理化性能参数比较 $[39]$

\begin{tabular}{|c|c|c|c|c|c|c|c|c|c|c|c|c|}
\hline \multirow[b]{2}{*}{ 样品名称 } & \multicolumn{4}{|c|}{ 元素分析结果/\% } & \multicolumn{5}{|c|}{ CPMAS ${ }^{13} \mathrm{C}$ NMR 结果 } & \multirow{2}{*}{$\begin{array}{l}\text { TOC } \\
/ \%\end{array}$} & \multirow{2}{*}{$\begin{array}{c}\mathrm{DOC}^{\mathrm{a})} \\
/ \%\end{array}$} & \multirow{2}{*}{$\begin{array}{c}\text { CEC/ } \\
\mathrm{cmol} \\
(+) \cdot \mathrm{kg}^{-1}\end{array}$} \\
\hline & $\mathrm{C}$ & $\mathrm{H}$ & $\mathrm{O}$ & $\mathrm{N}$ & $\begin{array}{c}\text { 脂肪族碳 } \\
(\delta 0 \sim 100) / \%(c\end{array}$ & $\begin{array}{l}\text { 芳香族碳 } \\
\text { S100 165)/\% }\end{array}$ & $\begin{array}{c}\text { 羧基碳 } \\
\delta 165 \sim 185) /\end{array}$ & $\begin{array}{c}\text { 醛、酮 } \\
/ \%(\delta 185 \sim 220) / \%\end{array}$ & $\begin{array}{l}\text { 疏水 } \\
\text { 系数 }\end{array}$ & & & \\
\hline 官厅腐殖酸 & 24.01 & 3.96 & 25.20 & 2.97 & 55.9 & 21.8 & 15.0 & 5.3 & 0.72 & 28.76 & 2.78 & 9.36 \\
\hline 天津腐殖酸 & 62.53 & 4.80 & 32.34 & - & 41.1 & 41.1 & 2.5 & 12.4 & 0.92 & 64.77 & 0.87 & 3.22 \\
\hline
\end{tabular}

a) 在 $0.005 \mathrm{~mol} / \mathrm{L} \mathrm{CaCl}_{2}$ 存在条件下测定

表 2 两种腐殖质中可溶解部分(溶解腐殖质)的 ${ }^{1} \mathrm{H}$ NMR分析结果比较 ${ }^{[39]}$

\begin{tabular}{|c|c|c|c|c|}
\hline 样品名称 & 脂肪族化合物 $(\delta 0 \sim 3.0) / \%$ & 芳香族化合物 $(\delta 6.5 \sim 8.5) / \%$ & 极性基团 $(\delta 3.0 \sim 6.5) / \%$ & 疏水系数 \\
\hline 官厅腐殖酸 & 24.6 & - & 75.4 & 0.33 \\
\hline 天津腐殖酸 & 15.4 & 0.5 & 84.1 & 0.19 \\
\hline
\end{tabular}




\section{2 腐殖酸在云母界面上的吸附条件实验}

将一定量腐殖酸样品加入到一定量的由 0.005 $\mathrm{mol} / \mathrm{L} \mathrm{CaCl}$ 和 $100 \mathrm{mg} / \mathrm{L} \mathrm{NaN}_{3}$ 组成的混合液中, 于磁 力摚拌器上摚拌, 缓慢加入 $0.1 \mathrm{~mol} / \mathrm{L} \mathrm{NaOH}$ 溶液, 调 溶液 $\mathrm{pH}$ 值为 9 左右, 使之慢慢溶解. 第二天继续加 入 $\mathrm{NaOH}$ 溶液, 调节溶液 $\mathrm{pH}$ 值为 9 左右, 将溶液摚 拌过夜, 静置 $24 \mathrm{~h}$, 将上清液取出, 盛放在棕色瓶中. 该溶液作为腐殖酸储备液, 于冰箱中 $\left(4^{\circ} \mathrm{C}\right)$ 保存, 备 用.

将腐殖酸储备液经稀释分别配制成 $10 \mathrm{mg} / \mathrm{L}$ 和 $100 \mathrm{mg} / \mathrm{L}$ 腐殖酸溶液(含 $0.005 \mathrm{~mol} / \mathrm{L} \mathrm{CaCl}$ 和 100 $\mathrm{mg} / \mathrm{L} \mathrm{NaN_{3 }}$, 且在 $\mathrm{pH}$ 为 8 9 的微碱性条件下), 置于 $50 \mathrm{~mL}$ 带塞磨口三角瓶中, 加入新解理的天然云母片, 室温下于摇床上振荡 $60 \mathrm{~h}$. 取出云母片, 用去离子水 漂洗, 自然晾干后进行原子力显微镜( AFM)成像观 察.

\section{3 有萗共吸附条件下腐殖酸在云母界面上的吸 附条件实验}

将上述制备好的腐殖酸储备溶液与菜饱和储备溶 液按适当比例混合、稀释，配制成含一定浓度 $(30 \mathrm{mg} / \mathrm{L})$ 的腐殖酸及菜溶液, 然后再加入新解理的天然云母片, 按第 1.2 小节所述操作步骤进行吸附条件实验, 同时 做空白对照. 吸附完成后, 将云母片取出, 经去离子 水漂洗、晾干, 在原子力显微镜下进行成像观察.

\section{4 腐殖酸在云母界面上吸附实验样品的原子力 显微镜观察}

将第 1.2 及 1.3 小节中的云母片样品用双面胶粘 在金属样品盘上，放到原子力显微镜的样品操作台 上, 用美国 DI 仪器公司 Nanoscope III a Multimode 原 子力显微镜, 采用接触模式在大气环境下进行成像 观察, 所用探针为商用 $\mathrm{V}-$ 型 $\mathrm{Si}_{3} \mathrm{~N}_{4}$ 探针, 微悬臂长度为 $200 \mu \mathrm{m}$, 力常数为 $0.12 \mathrm{~N} / \mathrm{m}$. 所有图象均在恒力模式 下获得. 扫描器采用 $\mathrm{E}$ 型压电陶瓷扫描管, 扫描参数 如扫描尺度、Setpoint 值、扫描速率、增益参数如 I-gain 及 $P$-gain 值等在成像扫描过程中根据实际情况进行 调节，并以使探针针尖加载到样品上的作用力最小 且能获得稳定扫描图像质量为依据，以防止因针尖
压力过大造成样品损坏及扫描图像因假象的存在而 失真等情况出现. 每一实验条件经反复实验确认，每 次制备两个平行样品, 每一样品至少进行 3 次不同位 置的 AFM 观测. 存储后的图像经 Nanoscope Image Analysis 分析处理软件进行平滑处理(必要时)后, 进 行形貌特征的截面分析(Section analysis)与测量.

\section{2 结果与讨论}

图 1 和 2 分别给出了在不同实验浓度条件下官厅 和天津两种腐殖质在纳米尺度上的界面吸附形态特 征的 AFM 图像比较. 可以看出, 两种腐殖质在微观 结构形态上存在着明显的差异. 官厅腐殖酸聚集体 呈不规则的链环状结构, 由平均宽度为 $40 \mathrm{~nm}$ 左右、 厚度约为 $0.5 \sim 1 \mathrm{~nm}$ 的高分子链围成一个个长宽不一 的细长闭环结构, 并有少量颗粒状形态夹杂其中; 而 天津腐殖质的结构则比较单一, 为致密的球形颗粒, 直径从 250 至 $330 \mathrm{~nm}$, 厚度 $8.6 \mathrm{~nm}$ 左右. 在较高浓度 (30 mg/L, $100 \mathrm{mg} / \mathrm{L}$, 图 2)的实验条件下, 两种腐殖质 都观察到呈多孔片层、球形或团簇颗粒状聚集体的微 观结构形态. 相对于官厅腐殖质而言, 天津腐殖质的 结构形态则变化较少, 且结构较致密, 它在云母表面 上聚集形成的自组装结构分布相对均匀，成膜性能 较好; 而官厅腐殖质形成的聚集体结构却比较疏松. 此结果正好与两种腐殖质的不同来源及化学组成分 析结果相吻合.

从表 1 和 2 可以看出, 官厅腐殖质具有较高的 $\mathrm{N}$ 含量(2.97\%), 而且 $\mathrm{O} / \mathrm{C}$ 较大 $(0.79), \mathrm{H} / \mathrm{C}(1.98)$ 大约等 于 2, CPMAS ${ }^{13} \mathrm{C}$ NMR 谱图在 $\delta=171$ 化学位移处存 在较强的羧基或胺碳峰, 脂肪族碳含量较高, 其溶解 有机碳含量远大于天津腐殖质样品,表明官厅腐殖质 中极性脂肪族物质含有很高, 且含有部分蛋白质氨 基酸等生物大分子物质; 而天津腐殖质样品中 $\mathrm{N}$ 含 量较低(未检出), 而 C 和 O 含量都很高(分别为 $62.53 \%$ 和 $32.34 \%), \mathrm{H} / \mathrm{C}$ 比(0.92)接近 1, CPMAS ${ }^{13} \mathrm{C}$ NMR 谱 图中羧酸或胺碳 $(\delta=175)$ 含量较低, 醛、酮(羰基碳, $\delta=204)$ 含量则相对较高, 疏水系数(0.92)接近 1, 芳 香族碳 $(41.1 \%)$ 与脂肪族碳( $41.1 \%)$ 含量基本相当. 由 于官厅腐殖质中含有较多的极性脂肪族物质，它们 具有类似表面活性剂的分子结构特征，在溶液中可 

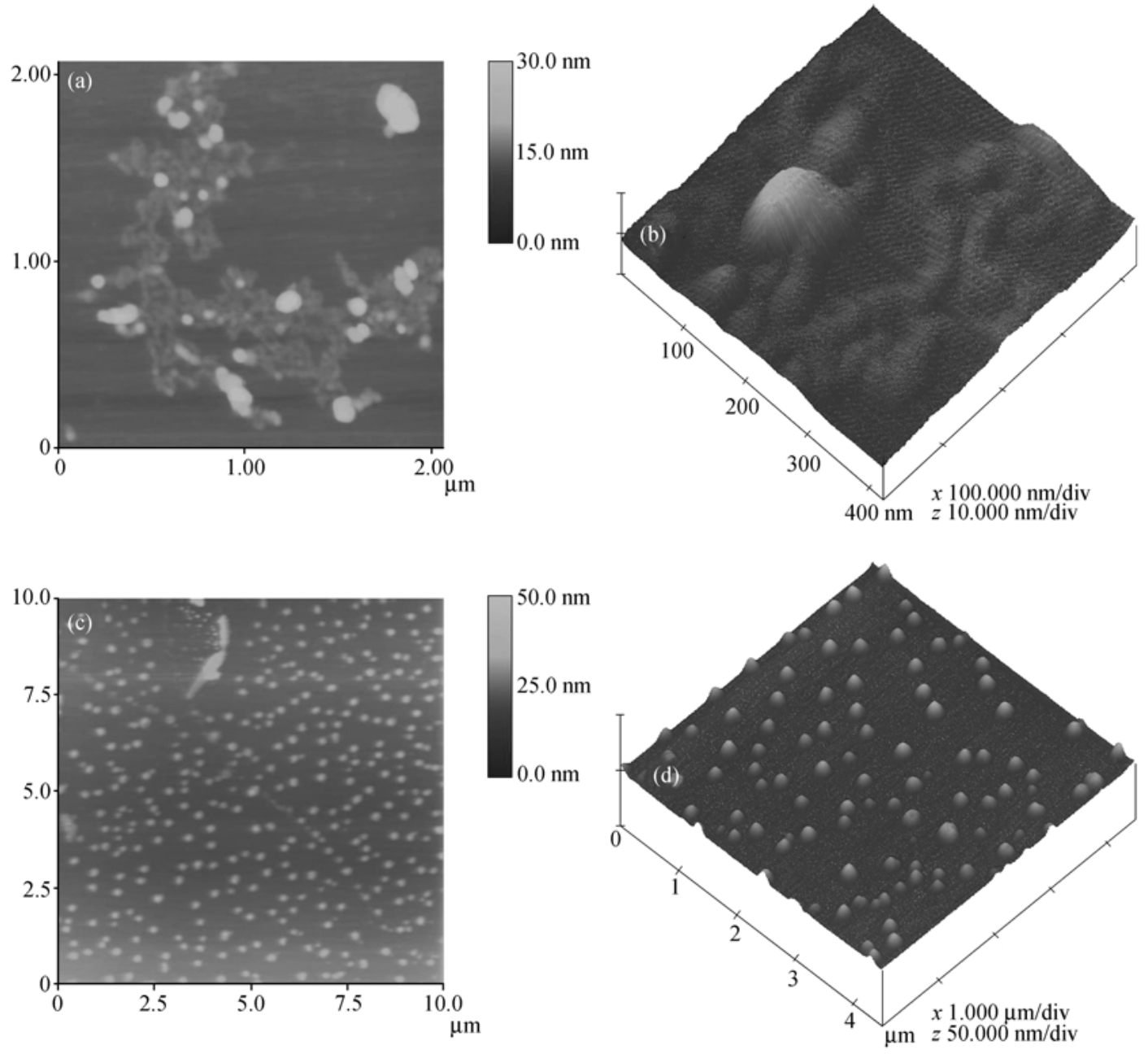

图 $110 \mathrm{mg} / \mathrm{L}$ 实验浓度条件下官厅和天津两种不同腐殖质样品在云母界面上吸附聚集的 AFM 图像比较 (a)和(b) 呈链环状结构的官厅腐殖质聚集体形貌及其局部放大三维立体图像; (c)和(d) 呈分散球形颗粒状的天津腐殖质聚集体形貌及其 局部放大三维立体图像

形成类胶束结构，其亲水、憎水组分/基团在界面上可 自组装成各种聚集结构. 除观察到多孔片层状结构、 颗粒状聚集体(由直径在 $50 \mathrm{~nm}$ 左右、厚度在 8 15 nm 的球形颗粒聚集成大小不一的椭球形状)等结构形态 外，官厅腐殖质还可观察到疏松海绵状、枝条状(宽度 从 45 到 $65 \mathrm{~nm}$ 不等)及凝胶团块状等更丰富的形貌结 构特征, 图 3 给出了官厅腐殖质在较高实验浓度条件 下所观测到的进一步实验结果.

为了深入了解腐殖质对疏水性有机污染物在天 然颗粒物界面上的吸附作用机理, 本文对有萗共吸 附实验条件下官厅腐殖质在云母界面上的微观结构
形态作了进一步的 AFM 成像观测. 图 4 给出了本实 验的观测结果. 可以看出, 有荎共吸附实验条件下, 官厅腐殖质的界面吸附形态发生了明显改变, 支链 状结构明显变窄, 多孔片层或海绵状结构变得更加致 密, 孔状结构趋向减少，并观察到类似凝胶团块状特 征形貌出现. 例如, 未加入菜时支链状聚集体形貌宽 度在 500 650 nm 左右, 多孔片层状聚集体形貌宽度为 $1.5 \mu \mathrm{m}$ 左右, 厚度平均为 $0.916 \mathrm{~nm}$, 空洞孔径在 40 到 $250 \mathrm{~nm}$ 不等或者更小; 有荎存在时支链宽度下降到 300 350 nm, 片层宽度为 $500 \mathrm{~nm}$ 左右, 厚度平均为 $1.217 \mathrm{~nm}$, 层中空洞孔径从 40 到 $100 \mathrm{~nm}$ 不等或者更 

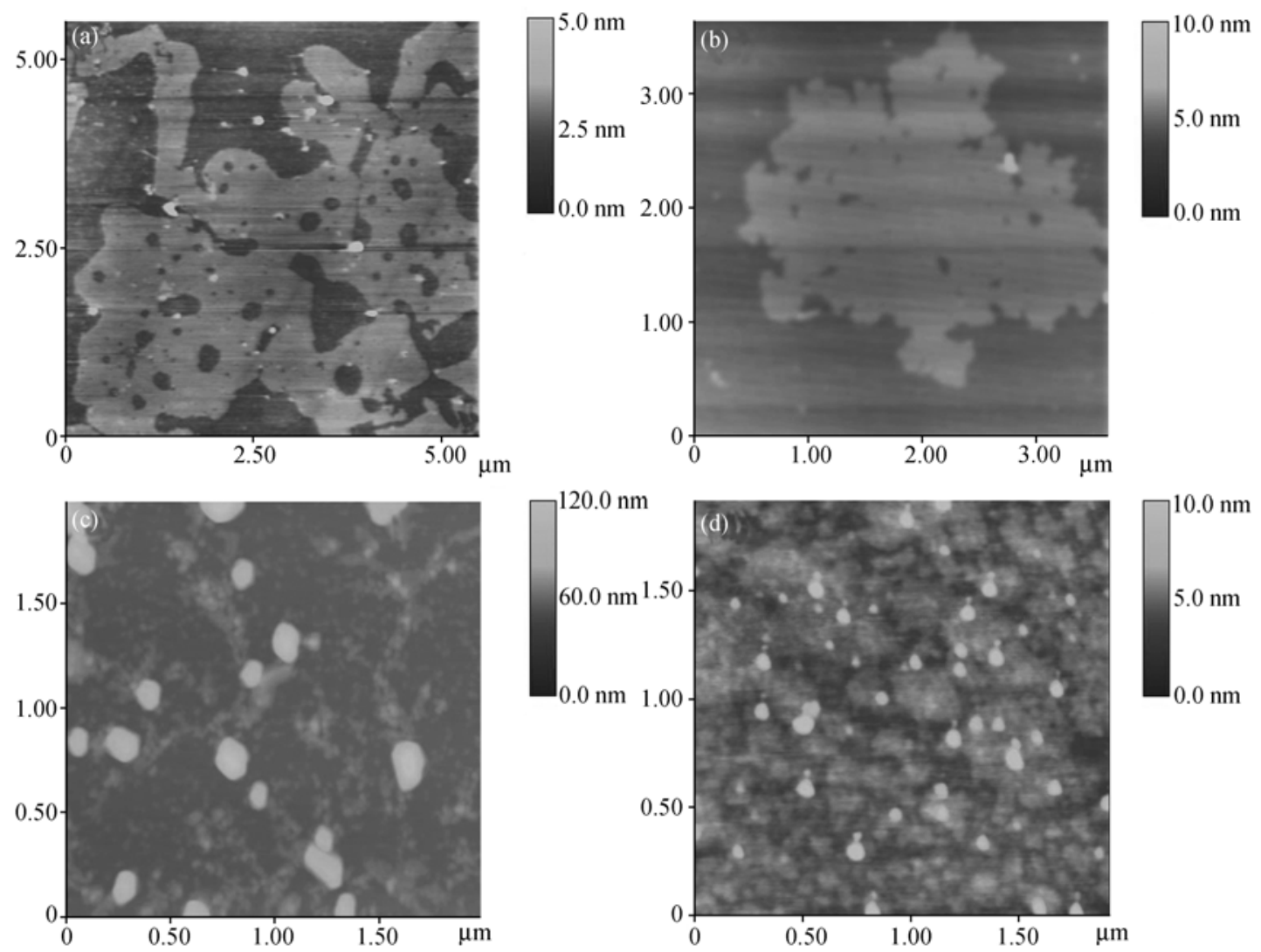

图 2 较高实验浓度((a), (b) $30 \mathrm{mg} / \mathrm{L}$ 及(c), (d) $100 \mathrm{mg} / \mathrm{L}$ ) 条件下官厅和天津两种腐殖质在云母界面上 吸附聚集形态的变化情况

(a)和(c)为官厅腐殖质形貌观测结果; (b)和(d)为天津腐殖质形貌观测结果

小. 因此，有荎共吸附实验条件下，官厅腐殖酸由多 孔状类似海绵的链环状聚集体向孔结构较少的片层 状聚集体结构转变, 表明菜分子与溶解腐殖质分子 发生了相互作用，并使腐殖质分子之间的联系进一 步加强, 从而结合更加紧密.

关于腐殖质的分子结构问题，虽然目前仍存在 着不少争论, 但是腐殖质的疏水区域和亲水区域已 被实验所证实 ${ }^{[20,41]}$. 腐殖质作为两性聚电解质, 具有 两性官能团, 有疏水与亲水两种性质, 哪种性质起主 导作用与溶液条件有关. 一般说来, 它既可吸附于颗 粒物表面, 也可独立存在于溶液中, 还可两种状态并 存. 其本身的形态呈链状还是团状等都随溶液条件 如腐殖酸浓度、 $\mathrm{pH}$ 、盐度或离子强度、高价金属离 子[16,34,35] 以及温度等多种因素而变, 并不是完全固定 的. 菜等疏水性有机物与腐殖质的疏水基团有亲和
力, 根据相似相溶原理, 它们吸附到颗粒物上也主要 是吸附到颗粒物的有机包围层上, 因而早期研 究 ${ }^{[8,9,42 \sim 45]}$ 认为是溶解或分配过程, 呈线性. 但后来研 究 $[10 \sim 13,46 \sim 55]$ 发现, 还普遍存在一定范围的非线性、延 迟和竞争现象(特别是在有机物浓度相对较低的情况 下), 吸附过程呈现出明显的 “两阶段” 性或 “双峰” 现象。例如, Weber及其合作者 ${ }^{[56]}$ 发现疏水性有机污 染物在颗粒物上的吸附是由起始时的快速吸附和随 后出现的缓慢吸附两个阶段构成的, 整个吸附过程需 要数周到几个月甚至几年才能达到平衡. 其原因有多 种解释，也可能有多种原因共存造成，有几何位阻因 素, 有官能团差异的化学因素, 造成动力学趋于平衡 过程中的非线性分配, 并提出了有机质结构形态的双 相理论模型. 此模型认为在土壤及沉积物吸附剂的有 机质中同时共存着两种性质截然不同的吸附区域: 

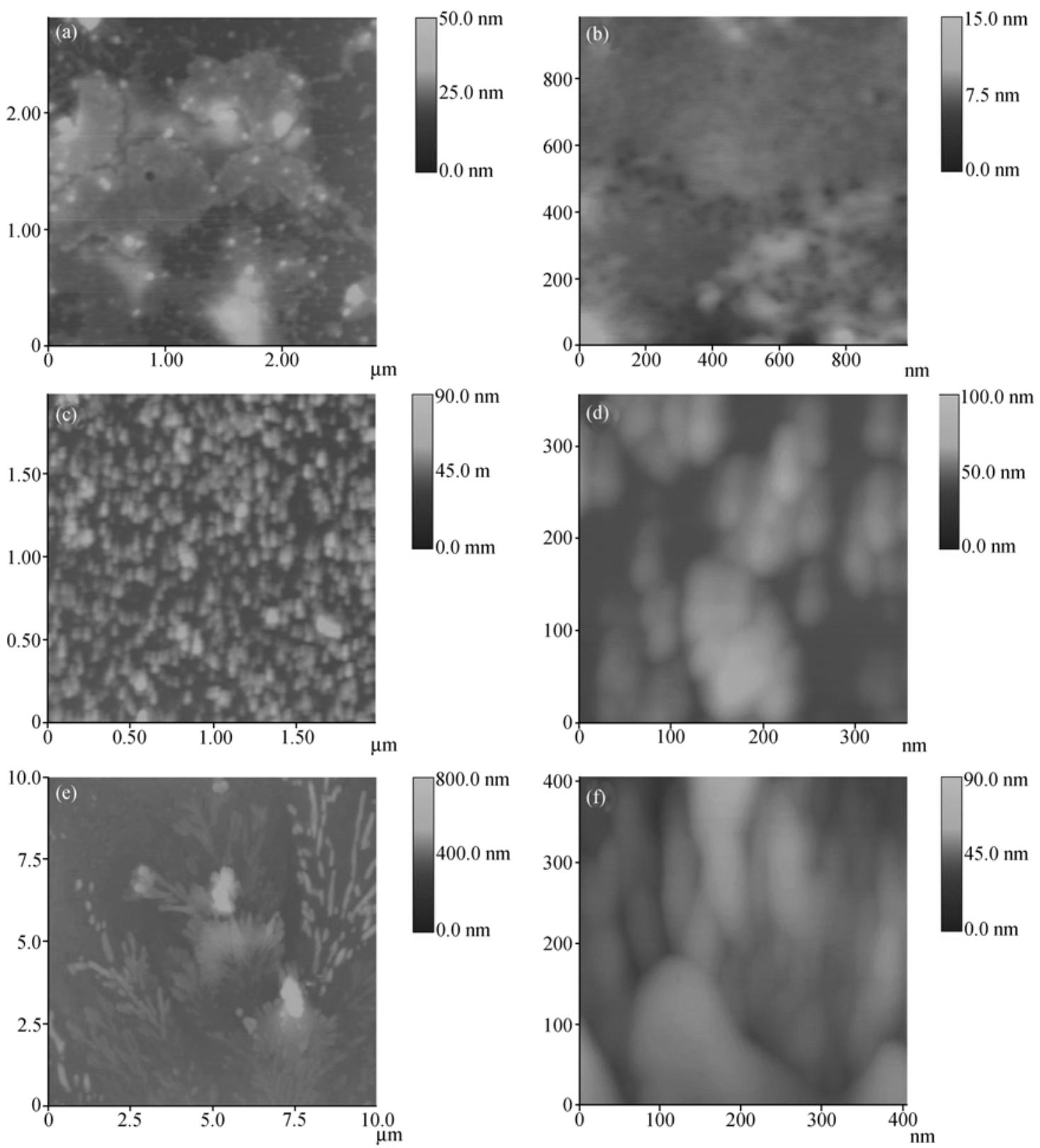

图 $3100 \mathrm{mg} / \mathrm{L}$ 实验条件下官厅腐殖质在云母界面上呈现的多分散结构特征

(a) 为凝胶团块状结构; (b) 为疏松海绵状结构; (c)和(d)为球状颗粒聚集体及其局部放大图像; (e)和(f)为枝条状聚集体及其局部放大结构

结构较疏松、呈无定型形态的 “分配” 吸附区域和结 构较致密的 “特殊位” 吸附区域. 但是, 到目前为止 还没有能完全区分和表征这两类有机质形态存在的 直接证据. 本文通过对官厅和天津两种不同来源及 组成的腐殖质样品微观形貌的原子力显微镜观察,
直接观测到溶解腐殖质所具有的不同的界面形态特 征，从而提供了有关腐殖质多相不均匀结构的初步 直接证据, 并对有萗共吸附实验条件下官厅腐殖质 界面吸附聚集形态的变化情况作了进一步 AFM 观测. 通过官厅腐殖质由疏松的多孔海绵状结构向比较致 

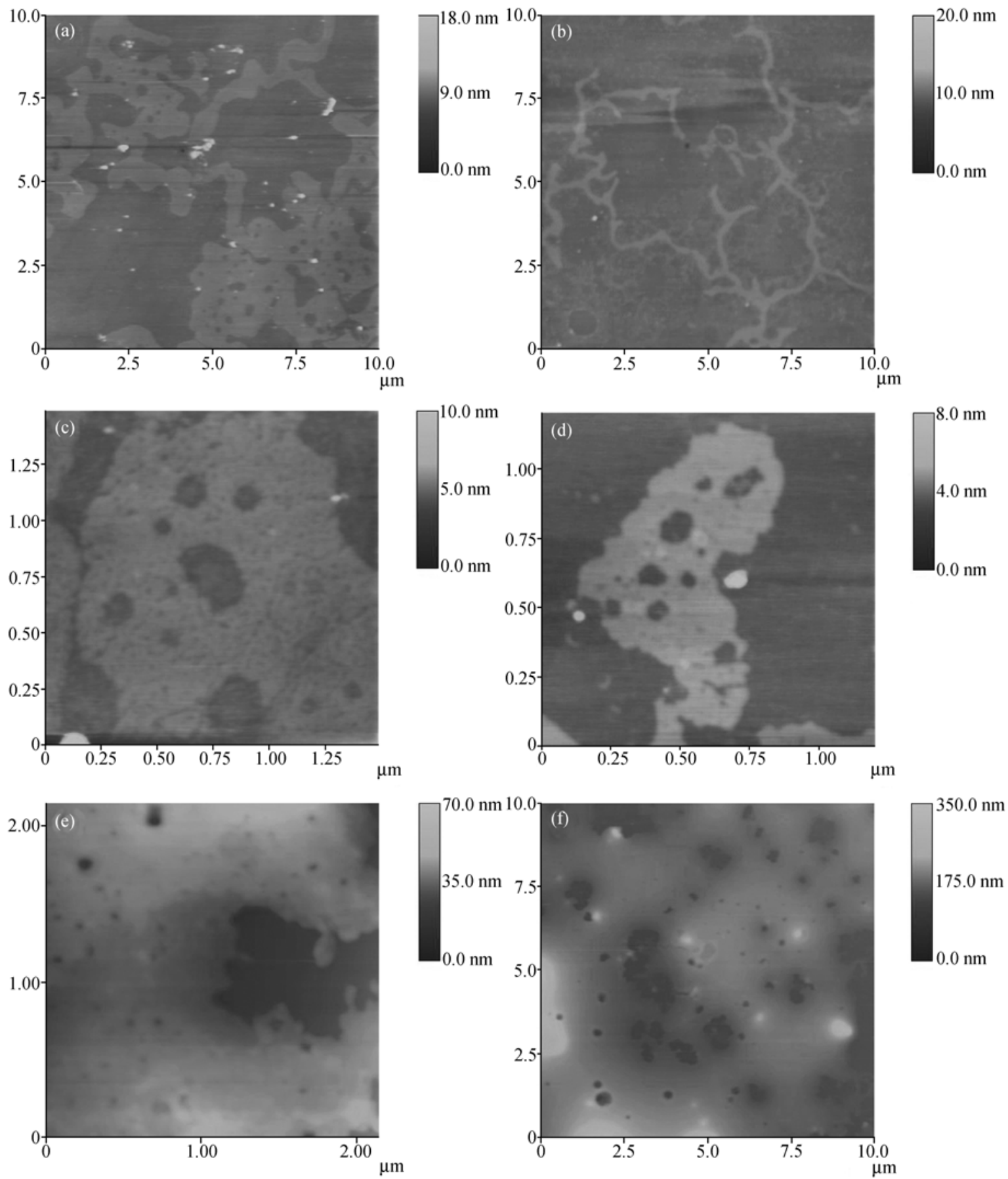

图 4 有荎共吸附实验条件下官厅腐殖质在云母界面上吸附聚集形态变化情况的 AFM 观测 (a), (c)和(e)为未加入荎的腐殖质对照空白的形貌图像; (b), (d) 和(e)为加入 $30 \mathrm{mg} / \mathrm{L}$ 傃条件下官厅腐殖质的微观形貌图像

密的片层结构过渡，初步表明了荎分子与溶解腐殖 质分子发生了相互作用，并使腐殖质分子之间的联 系进一步加强，结合更加紧密．同时，官厅腐殖质多
孔链环状结构中心有可能作为腐殖质分子的疏水性 吸附区域，通过疏水作用与荎分子发生吸附，从而促 进荎分子在界面上的吸附和聚集. 


\section{3 结论}

直接观测到官厅和天津两种不同来源和组成的 腐殖质样品的微观形貌存在明显差异, 并与其官能 才组成及存在条件紧密相关. 与天津腐殖质相比, 官 厅腐殖质的聚集体结构较疏松, 且非均相、多分散结 构特征更加明显, 这可能与官厅腐殖质中含有较多 的极性物质有关.

有萗共吸附条件下，官厅腐殖质的界面形态发 生明显改变, 支链状结构变窄, 多孔片层状结构变得 更加致密，孔结构趋向减少，表明萗分子与溶解腐殖 质分子发生了相互作用，并使腐殖质分子之间的联 系进一步加强，结合更加紧密. 同时，官厅腐殖质多 孔链环状结构中心有可能作为腐殖质分子的疏水性 吸附区域，通过疏水作用与萗分子发生吸附，从而促 进萗分子在界面上的吸附和聚集.

\section{参考文献}

1 Hayes M H B, Clapp C E. Humic substances: Considerations of compositions, aspects of structure, and environmental influences. Soil Science, 2001, 166(11): 723 737[DOI]

2 MacCarthy P. The principles of humic substances. Soil Science, 2001, 166(11): 738 751[DOI]

3 Swift R S. Macromolecular properties of soil humic substances: Fact, fiction, and opinion. Soil Science, 1999, 164(11): 790 802[DOI]

4 Wershaw R L. Molecular aggregation of humic substances. Soil Science, 1999, 164(11): 803 813[DOI]

5 Tombacz E. Colloidal properties of humic acids and spontaneous changes of their colloidal state under variable solution conditions. Soil Science, 1999, 164(11): 814 824[DOI]

6 Garbarini D R, Lion L W. Influence of the nature of soil organics on the sorption of toluene and trichloroethylene. Environ Sci Technol, 1986, 20(12): 1263 1269[DOI]

7 Gauthler T D, Seitz W R, Grant C L. Effects of structural and compositional variations of dissolved humic materials on pyrene $K_{\text {OC }}$ values. Environ Sci Technol, 1987, 21(3): 243 248[DOI]

8 Chiou C T, Malcolm R L, Brinton T I, et al. Water solubility enhancement of some organic pollutants and pesticides by dissolved humic and fulvic acids. Environ Sci Technol, 1986, 20(5): 502 508[DOI]

9 Chiou C T, Kile D E, Brinton T I, et al. A comparison of water solubility enhancements of organic solutes by aquatic humic materials and commercial humic acids. Environ Sci Technol, 1987, 21(12): 1231 1234[DOI]

10 Rutherford D W, Chiou C T, Kile D E. Influence of soil organic matter composition on partition of organic compounds. Environ
Sci Technol, 1992, 26(2): 336 340[DOI]

11 Grathwohl P. Influence of organic matter from soils and sediments from various origins on the sorption of some chlorinated aliphatic hydrocarbons: Implications on $K_{\mathrm{OC}}$ correlations. Environ Sci Technol, 1990, 24(11): 1687 1693[DOI]

12 Murphy E M, Zachara J M, Smith S C, et al. Interaction of hydrophobic organic compounds with mineral-bound humic substances. Environ Sci Technol, 1994, 28(7): 1291 1299

13 Huang W L, Weber W J Jr. A distributed reactivity model for sorption by soils and sediments. 10. Relationships between desorption, hysteresis, and the chemical characteristics of organic domains. Environ Sci Technol, 1997, 31(9): 2562 2569[DOI]

14 Tipping E, Higgins D C. The effect of adsorbed humic substances on the colloid stability of haematite particles. Colloids Surf, 1982, 5(2): 85 92[DOI]

15 Wilkinson K J, Nègre J-C, Buffle J. Coagulation of colloidal material in surface waters: the role of natural organic matter. J Contam Hydrol, 1997, 26(1-4): 229 243[DOI]

16 Engebretson R R, von Wandruszka R. Kinetic aspects of cation-enhanced aggregation in aqueous humic acids. Environ Sci Technol, 1998, 32(4): 488 493[DOI]

17 Ghosh K, Schnitzer M. Macromolecular structures of humic substances. Soil Science, 1980, 129(5): 266 276

18 Camerson R S, Thomton B K, Swift R S, et al. Molecular weight and shape of humic acid from sedimentation and diffusion measurements on fractionated extracts. Journal of Soil Science, 1972, 23, 394 408

19 Cornel P K, Summers R S, Roberts P V. Diffusion of humic acid in dilute aqueous solution. $\mathrm{J}$ of Colloid and Interface Science, 1986, 110(1): 149 164[DOI]

20 Engebretson R R, von Wandruszka R. Microorganization in dissolved humic acids. Environ Sci Technol, 1994, 28(11): 1934 1941[DOI]

21 Lobartini J C, Tan K H. Differences in humic acid characteristics as determined by carbon-13 nuclear magnetic resonance, scanning electron microscopy, and infrared analysis. Soil Sci Soc Am J, 1988, 52(1): 125 130

22 Hatcher P G, Dria K J, Kim Sunghwan, et al. Modern analytical studies of humic substances. Soil Sci, 2001, 166(11): 770 794[DOI]

23 Zhou Y M, Liu R X, Tang H X. Kinetics study of aqueous sorption of phenanthrene to humic acids and sediments. J of Environ Sci, 2004, 16(3): 408 413

24 Chen Y, Schnitzer M. Scanning electron microscopy of a humic acid and of a fulvic acid and its metal and clay complexes. Soil Sci Soc Am J, 1976, 40(5): 682 686

25 Stevenson I L, Schnitzer M. Transmission electron microscopy of extracted flvic and humic acids. Soil Science, 1982, 133(3): 179 185

26 Paredes J I, Martínez-Alonso A, Tascón J M D. Application of scanning tunneling and atomic force microscopies to the characterization of microporous and mesoporous materials. Microporous 
and Mesoporous Materials, 2003, 65(2-3): 93 126[DOI]

27 Heil D, Sposito G. Organic matter role in illitic soil colloids flocculation: III. Scanning force microscopy. Soil Sci Soc Am J, 1995, 59(1): 266 269

28 Gerin P A, Dufrêne Y F. Native surface structure of natural soil particles determined by combining atomic force microscopy and X-ray photoelectron spectroscopy. Colloids and Surfaces B, 2003, 28(4): 295 305[DOI]

29 Namjesnik-Dejanovic K, Maurice P A. Atomic force microscopy of soil and stream fulvic acids. Colloids and Surfaces A, 1997, 120(1-3): 77 86[DOI]

30 Maurice P A, Namjesnik-Dejanovic K. Aggregate structures of sorbed humic substances observed in aqueous solution. Environ Sci Technol, 1999, 33(9): 1538 1541[DOI]

31 Wilkinson K J, Balnois E, Leppard G G, et al. Characteristic features of the major components of freshwater colloidal organic matter revealed by transmission electron and atomic force microscopy. Colloids Surf A, 1999, 155 (2-3): 287 310[DOI]

32 Balnois E, Wilkinson K J, Lead J R, et al. Atomic force microscopy of humic substances: Effects of $\mathrm{pH}$ and ionic strength. Environ Sci Technol, 1999, 33(21): 3911 3917[DOI]

33 Plaschke M, Römer J, Klenze R, et al. In situ AFM study of sorbed humic acid colloids at different $\mathrm{pH}$. Colloids and Surfaces A, 1999, 160(3): 269 279[DOI]

34 Plaschke M, Römer J, Klenze R, et al. Influence of europium(III) on the adsorption of humic acid onto mica studied by AFM. Surf Interface Anal, 2000, 30(1): 297 300. [DOI]

35 Plaschke M, Rothe J, Schäfer T, et al. Combined AFM and STXM in situ study of the influence of $\mathrm{Eu}(\mathrm{III})$ on the agglomeration of humic acid. Colloids Surf A, 2002, 197(1-3): 245 256[DOI]

36 Liu Aiguo, Wu R C, Eschenazi E, et al. AFM on humic acid adsorption on mica. Colloids and Surfaces A, 2000, 174(1-2): 245 252[DOI]

37 刘 燕，王越兴，莫华娟，等. 有机底物对活性污泥胞外聚合物 的影响. 环境化学, 2004, 23(3): 252 257

38 Schnitzer M. Organic matter extraction. In: Page A L, ed. Methods of Soil Analysis. Part 2. 2nd ed. Madison: American Society of Agronomy, 1982. 581

39 周岩梅. 多环芳烃有机物在天然颗粒物界面上的吸附机理研究. 北京：中科院生态环境研究中心博士论文, 2003.

40 Hendershot W H, Duquette M. A simple barium chloride method for determining cation exchange capacity and exchangeable cations. Soil Sci Soc Am J, 1986, 50: 605 608

41 Conte P, Piccolo A. Conformational arrangement of dissolved humic substances: influence of solution composition on association of humic molecules. Environ Sci Technol, 1999, 33(10): 1682 $1690[$ DOI]
42 Karickhoff S W, Brown D S, Scott T A. Sorption of hydrophobic pollutants on natural sediments. Water Res, 1979, 13(3): 241 248

43 Chiou C T, Porter P E, Schmedding D W. Partition equilibria of nonionic organic compounds between soil organic matter and water. Environ Sci Technol, 1983, 17(4): 227 231[DOI]

44 Kile D E, Chiou C T, Zhou H D, et al. Partition of nonpolar organic pollutants from water to soil and sediment organic matters. Environ Sci Technol, 1995, 29(5): 1401 1406[DOI]

45 Chiou C T, Mcgroddy S E, Kile D E. Partition characteristics of polycyclic aromatic hydrocarbons on soils and sediments. Environ Sci Technol, 1998, 32(2): 264 269[DOI]

46 Chiou C T, Kile D E. Deviations from sorption linearity on soils of polar and nonpolar organic compounds at low relative concentrations. Environ Sci Technol, 1998, 32(3): 338 343[DOI]

47 Kile D E, Wershaw R T, Chiou C T. Correlation of soil and sediment organic matter polarity to aqueous sorption of nonionic compounds. Environ Sci Technol, 1999, 33(12): 2053 2056[DOI]

48 Chiou C T, Kile D E, Rutherford D W, et al. Sorption of selected organic compounds from water to a peat soil and its humic acid and humin fractions: potential sources of the sorption nonlinearlity. Environ Sci Technol, 2000, 34(7): 1254 1258[DOI]

49 Zhu L Z, Chen B L, Tao S, et al. Interaction of organic contaminants with mineral-adsorbed surfactants. Environ Sci Technol, 2003, 37(17): 4001 4006[DOI]

50 Murphy E M, Zachara J M, Smith S C. Influence of mineral-bound humic substances on the sorption of hydrophobic organic compounds. Environ Sci Technol, 1990, 24(10): 1507 1516[DOI]

51 McGinley P M, Katz L E, Weber W J Jr. A distributed reactivity model for sorption by soils and sediments. 2. Multicomponent systems and competitive effects. Environ Sci Technol, 1993, 27(8): 1524 1531[DOI]

52 Haggerty R, Gorelick S M. Multiple-rate mass transfer for modeling diffusion and surface reactions in media with pore-scale heterogeneity. Water Resour Res, 1995, 31(10): 2383 2400[DOI]

53 Xia G S, Ball W P. Adsorption-partitioning uptake of nine low-polarity organic chemicals on a natural sorbent. Environ Sci Technol, 1999, 33(2): 262 269[DOI]

54 Xia G S, Ball W P. Polanyi-based models for the competitive sorption of low-polarity organic contaminants on a natural sorbent. Environ Sci Technol, 2000, 34(7): 1246 1253[DOI]

55 Xia G S, Pignatello J J. Detailed sorption isotherms of polar and apolar compounds in a high-organic soils. Environ Sci Technol, 2001, 35(1): 84 94[DOI]

56 Weber W J Jr, Huang W L. A distributed reactivity model for sorption by soils and sediments. 4. Intraparticle heterogeneity and phase-distribution relationships under nonequilibrium conditions. Environ Sci Technol, 1996, 30(3): 881 888[DOI] 